\title{
Inflammatory bowel disease and risk of mortality in COPD
}

\author{
Maria Vutcovici ${ }^{1}$, Alain Bitton ${ }^{1,2}$, Pierre Ernst ${ }^{2,3}$, Abbas Kezouh $^{3}$, Samy Suissa ${ }^{2,3}$ \\ and Paul Brassard ${ }^{2,3}$ \\ Affiliations: \\ ${ }^{1}$ Division of Gastroenterology, McGill University Health Centre, Montréal, QC, Canada. \\ ${ }^{2}$ Faculty of Medicine, McGill University, Montréal, QC, Canada. \\ ${ }^{3}$ Lady Davis Institute for Medical Research, Jewish General Hospital, Montréal, QC, Canada.
}

\section{Correspondence:}

Paul Brassard, Centre for Clinical Epidemiology, Lady Davis Research Institute for Medical Research, Jewish General Hospital, 3755 Cote Ste-Catherine, H-4.24, Montréal, Québec H3T 1E2, Canada.

E-mail: paul.brassardamcgill.ca

ABSTRACT Patients with chronic obstructive pulmonary disease (COPD) have higher incidence and prevalence of other chronic inflammatory diseases, including inflammatory bowel disease (IBD). We assessed whether IBD onset increases mortality risk in patients with COPD or asthma-associated COPD.

Two population-based cohorts of COPD and asthma-COPD subjects were identified using the administrative health databases in Québec, Canada, 1990-2007. Death records were retrieved from the death certificate registry. Cox proportional hazards models were used to assess the impact of newly developed IBD on mortality risk.

The COPD and asthma-COPD cohorts included 273208 and 26575 patients, respectively, of which 697 and 119 developed IBD. IBD increased the risk of all-cause mortality in both COPD (hazard ratio 1.23, 95\% CI 1.09-1.4) and asthma-COPD (hazard ratio 1.65, 95\% CI 1.23-2.22). In asthma-COPD patients, IBD increased the risk of mortality from respiratory conditions (hazard ratio 2.18, 95\% CI 1.31-3.64); in COPD patients, IBD increased the risk of death from digestive conditions (hazard ratio 4.45, 95\% CI 2.39-8.30).

IBD is a risk factor for mortality in patients with pre-existing COPD or asthma-COPD. IBD increased mortality by respiratory and digestive conditions in patients with asthma-COPD and COPD, respectively.

@ERSpublications

IBD is a risk factor for mortality in patients with pre-existing COPD or asthma-associated COPD http://ow.ly/X6PaY 


\section{Introduction}

Chronic obstructive pulmonary disease (COPD) is a respiratory condition with a major public health impact, affecting about 210 million people worldwide [1] and ranked in the top five of the most frequent cause of death $[1,2]$. COPD is the major contributor to increases in deaths observed in high-income countries $[1,3]$. Only a third of all deaths in COPD patients are estimated to result from progressive respiratory failure [4]; therefore, other factors likely play an important part.

Approximately $20 \%$ of COPD patients present with a mix of asthma and COPD features [5-7], and there is evidence supporting an increased risk of morbidity, and possibly of comorbidity and mortality in such patients compared with those with COPD alone [5, 7].

The association with comorbid conditions may also impact COPD mortality [8]. While the association with neoplasms and cardiovascular diseases has been extensively explored, along with the role of cigarette smoke and systemic inflammation in the pathogenesis of these conditions, other comorbid conditions have received far less attention. COPD has been shown to be associated with other chronic inflammatory diseases, such as inflammatory bowel disease (IBD) $[9,10]$. Epidemiological studies have shown significant increases in incidence [11] and prevalence $[12,13]$ of IBD in patients with airway diseases. Furthermore, among IBD patients, mortality due to respiratory conditions in general [14] and COPD in particular [15] was found to be increased. To date, it is not known whether IBD may, in turn, impact COPD mortality. Therefore, we sought to assess whether new-onset IBD may constitute a risk factor for mortality among patients with COPD or asthma associated COPD.

\section{Methods}

\section{Data source}

The study was conducted using the healthcare administrative databases of the Régie de l'Assurance Maladie du Québec (RAMQ) and linked records from the death certificates registry provided by the Institut de la Statistique du Québec (Canada). The healthcare databases consist of routinely collected data regarding hospitalisations, procedures, outpatient physician visits, prescription claims filed under the provincial public drug plan as well as patient demographics. The public drug plan covers over $40 \%$ of the Québec population, including all residents aged 65 years and older, residents receiving social assistance and their dependents, and all residents without a private drug plan. The accuracy and comprehensiveness of the prescription claims data were evaluated, and the RAMQ prescriptions database was found to be one of the most accurate means to assess drugs dispensed to individuals in Québec [16].

\section{Study design and population}

In this population-based retrospective cohort study the source population consisted of all Québec residents who received one or more prescriptions for a specific respiratory medication from 1990 to 2007 . The respiratory medications included: any form of $\beta$-agonist, theophylline, ipratropium bromide, tiotropium, sodium cromoglycate, nedocromil, ketotifen, leukotriene antagonists (montelukast and zafirlukast) and inhaled corticosteroids. A base population of airway disease subjects was identified as all individuals with three or more prescriptions for a respiratory medication in any 1-year period and on at least two different dates. The cohort entry was considered the date of the third prescription for a respiratory medication. These criteria were successfully employed for identification of subjects with airway diseases in several RAMQ database studies addressing IBD incidence, risk of fractures, cataracts, glaucoma, tuberculosis or pneumonia [11, 17-22].

Due to the differences in disease characteristics described in patients with associated asthma features [5-7], two mutually exclusive cohorts of subjects with COPD and asthma-COPD were subsequently selected from the base population of individuals with airway diseases. The COPD cohort included all individuals aged 41 years or older at cohort entry for whom no record of asthma as principal diagnosis on a hospital discharge was identified in the database and no prescription was ever dispensed for any of the following medications: leukotriene antagonists (montelukast and zafirlukast), sodium cromoglycate, nedocromil or ketotifen. The selection criteria for the COPD cohort were consistent with our previous work addressing the incidence of IBD in COPD patients [11]. The asthma-COPD cohort included the individuals in the base population aged 41 years or older at cohort entry who did not satisfy the inclusion criteria for the COPD cohort.

\section{IBD patient identification}

We identified all subjects with new-onset IBD (either Crohn's disease or ulcerative colitis) occurring after entry in the COPD and asthma-COPD cohorts using an algorithm developed and validated in Alberta, Canada [23]. IBD patients were defined as the subjects with at least four physician billings in a 2-year interval or one hospital discharge with a principal diagnosis of Crohn's disease or ulcerative colitis. 
A 2-year IBD-free period prior to the Crohn's disease or ulcerative colitis patient-defining period was also applied as an additional condition to ensure incident IBD patients were captured. The identified subjects were considered as IBD patients at the time they fulfilled the algorithm criteria, whether that occurred at the time of the hospitalisation or at the fourth physician billing. The methodological approach is consistent with our previous work $[11,24]$.

\section{Assessment of mortality}

The death registry database contains the diagnostic code listed as the initial cause of death on the death certificates and up to 25 diagnostic codes for secondary causes of death. For the assessment of cause-specific mortality, the death counts were computed using the diagnostic code listed as the initial cause of death. Depending on the date of death, the diagnostic codes were classified according to International Classification of Diseases (ICD) revision 9 (prior to April 1, 2006) or 10 (April 2006 to December 2007). The following ICD codes were used in the analyses: J00-J99 (ICD-10) and 460-519 (ICD-9) for respiratory conditions; K00-K93 (ICD-10) and 520-579 (ICD-9) for digestive conditions; C00-D48 (ICD-10) and 140-239 (ICD-9) for neoplasms; I00-I99 (ICD-10) and 390-459 (ICD-9) for circulatory conditions.

\section{Statistical analysis}

Descriptive statistics were used to characterise the subjects making up the COPD and asthma-COPD cohorts. The impact of newly developed IBD on mortality was assessed using Cox proportional hazards models. The exposure, the presence of IBD, was modelled as a time-dependent variable. All the covariates used for model adjustment were assessed at cohort entry (baseline). These covariates included age at cohort entry, and use of antibiotics, anticoagulants, antihypertensives, antidiabetic and antirheumatic drugs, $\beta$-agonists, bronchodilators, cardiotropes, oral and inhaled corticosteroids, nonsteroidal anti-inflammatory drugs, nervous system-specific drugs, and vasodilators in the year prior to cohort entry. The number of hospitalisations in the year prior to cohort entry was also included as a covariate in the adjusted models as a marker of severity of comorbidities. Data analysis was performed using SAS/STAT ${ }^{\circ}$ software (version 9.3, SAS for Windows; SAS Institute, Cary, NC, USA). Ethical approval for this study was granted by the Research Ethics Committee of the Sir Mortimer B. Davis Jewish General Hospital (Montreal, Québec, Canada).

\section{Results}

A total of 273208 subjects fulfilled the selection criteria for the COPD cohort and 26575 for the asthma-COPD cohort, providing 1753986 and 219409 person-years of follow-up. There were 697 subjects who developed IBD after a mean \pm SD follow-up of $4.63 \pm 4.31$ years in the COPD cohort; 119 subjects developed IBD after a mean \pm SD follow-up of $5.40 \pm 4.03$ years in the asthma-COPD cohort. Table 1 presents the characteristics at cohort entry for the subjects with COPD or asthma-COPD stratified by the presence or absence of IBD during the follow-up. In the COPD cohort, subjects with IBD were less likely to have more than three hospitalisations and to have used anticoagulants, bronchodilators and cardiotropes in the year prior to cohort entry compared with subjects who never developed IBD; also, they were more likely to have used antirheumatics and nonsteroidal anti-inflammatory drugs. In the asthma-COPD cohort, subjects with IBD were less likely to have used anticoagulants, bronchodilators and inhaled corticosteroids in the year prior to cohort entry compared with those without IBD. Asthma-COPD subjects with IBD were more likely to have been hospitalised more than three times in the year prior to cohort entry than those who did not develop IBD.

The results for the association between IBD and all-cause mortality are presented in table 2 . There were a total of 123125 deaths in the COPD cohort, of which 243 occurred in the subjects who developed IBD. In the asthma-COPD cohort there were 8231 deaths, of which 45 were in subjects with IBD. There was an increased risk of mortality associated with developing IBD in both COPD (hazard ratio 1.23, 95\% CI 1.09-1.4) and asthma-COPD cohorts (hazard ratio $1.65,95 \%$ CI 1.23-2.22). The analysis stratified by type of IBD (table 3) revealed that Crohn's disease was associated with an increased risk of mortality among asthma-COPD patients (hazard ratio 1.78, 95\% CI 1.15-2.74); ulcerative colitis significantly increased the risk of mortality in both COPD (hazard ratio 1.27, 95\% CI 1.07-1.5) and asthma-COPD patients (hazard ratio 1.55, 95\% CI 1.03-2.32).

The most common causes of death in both COPD and asthma-COPD cohorts were, in order of frequency, circulatory conditions, neoplasms, respiratory and digestive conditions. There was an increased risk of mortality due to digestive conditions in the COPD subjects with IBD (hazard ratio 4.45, 95\% CI 2.39-8.3) (table 4). No increase in mortality risk for neoplasms, respiratory or circulatory conditions was associated with development of IBD in COPD subjects. Among asthma-COPD subjects, the presence of IBD was associated with an increased risk of mortality due to respiratory conditions (hazard ratio 2.18, 95\% CI 1.31-3.64) (table 5). As only one death by digestive conditions occurred in asthma-COPD subjects with 
TABLE 1 Characteristics of chronic obstructive pulmonary disease (COPD) and asthma-COPD patients at cohort entry

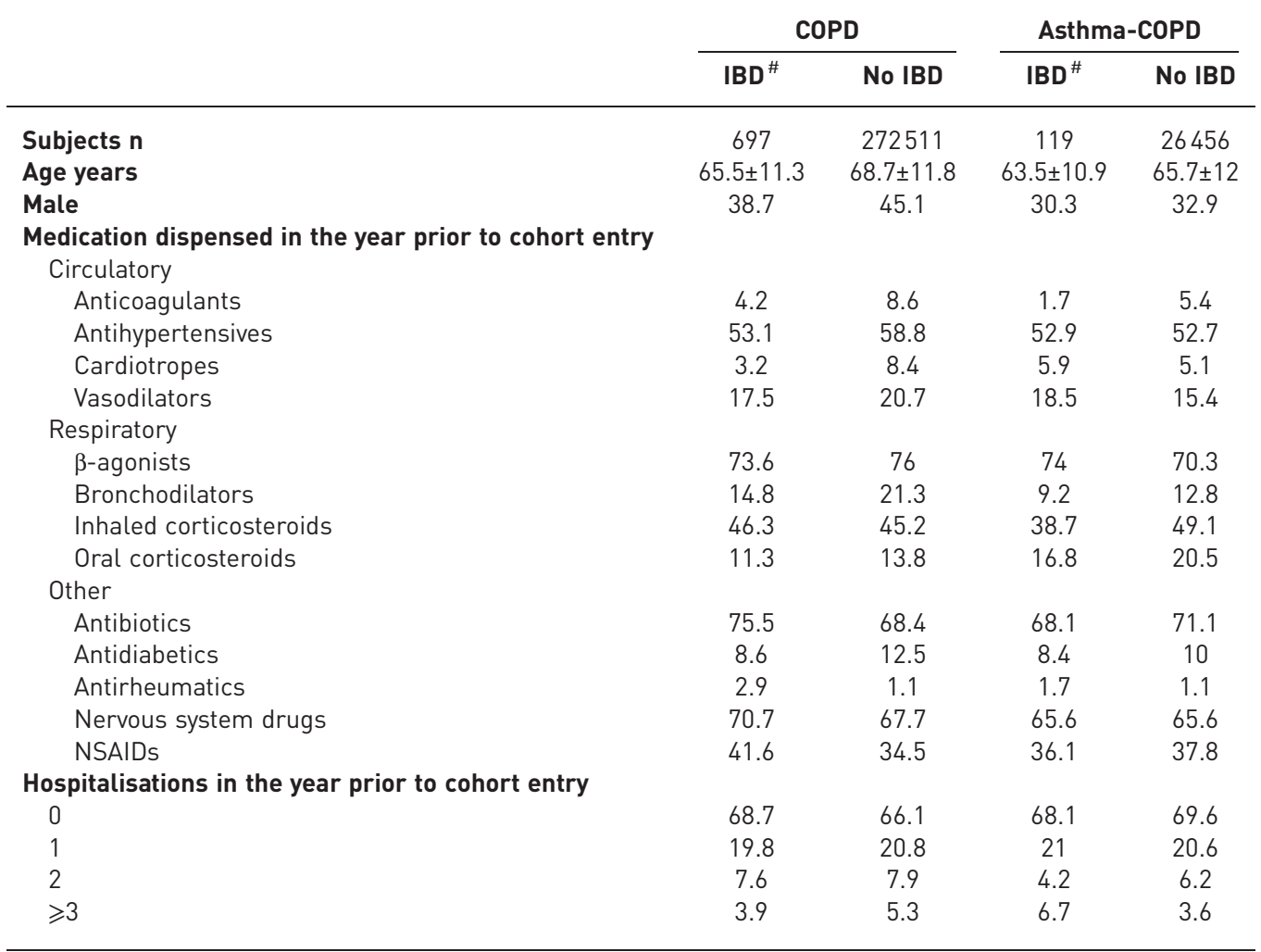

Data are presented as mean \pm SD or $\%$, unless otherwise stated. IBD: inflammatory bowel disease; NSAID: nonsteroidal anti-inflammatory drug. " : subjects IBD-free at cohort entry who develop IBD during the course of follow-up.

IBD, the risk of digestive conditions mortality was not assessed. The hazard ratios for the association between IBD and mortality due to neoplasms and circulatory conditions did not show any increase in risk of mortality in the asthma-COPD cohort. Also, we found no increase in risk of mortality from lung cancer or digestive cancers in either COPD or asthma-COPD subjects with IBD (data not shown). A stratification of cause-specific analyses by type of IBD was not performed due to the small number of deaths.

\section{Discussion}

In this large population-based study we found that new-onset IBD is a risk factor for all-cause mortality in patients with COPD or asthma-associated COPD. Both Crohn's disease and ulcerative colitis were associated with an increased mortality risk among asthma-COPD patients, while ulcerative colitis also impacted mortality among COPD patients. The risk of mortality due to respiratory conditions among

TABLE 2 Crude and adjusted hazard ratios for the association between inflammatory bowel disease (IBD) and all-cause mortality in patients with chronic obstructive pulmonary disease (COPD) and asthma-COPD

\begin{tabular}{|c|c|c|c|c|c|c|}
\hline & Patients & Deaths & $\begin{array}{l}\text { Total follow-up } \\
\text { (person-years) }\end{array}$ & $\begin{array}{c}\text { Mortality (deaths } \\
\text { per } 100 \text { person-years) }\end{array}$ & $\begin{array}{c}\text { Crude } \\
\text { hazard ratio }\end{array}$ & $\begin{array}{l}\text { Adjusted hazard } \\
\text { ratio }(95 \% \mathrm{CI})\end{array}$ \\
\hline \multicolumn{7}{|l|}{ COPD } \\
\hline IBD & 697 & 243 & 2928 & 8.30 & 1.14 & $1.23(1.09-1.4)$ \\
\hline \multicolumn{7}{|c|}{ Asthma-COPD } \\
\hline No IBD & 26456 & 8186 & 218827 & 3.74 & 1.00 & 1.00 (Reference) \\
\hline
\end{tabular}

Data are presented as $n$, unless otherwise stated. 
TABLE 3 Crude and adjusted hazard ratios for the association between Crohn's disease and ulcerative colitis and all-cause mortality in patients with chronic obstructive pulmonary disease (COPD) and asthma-COPD

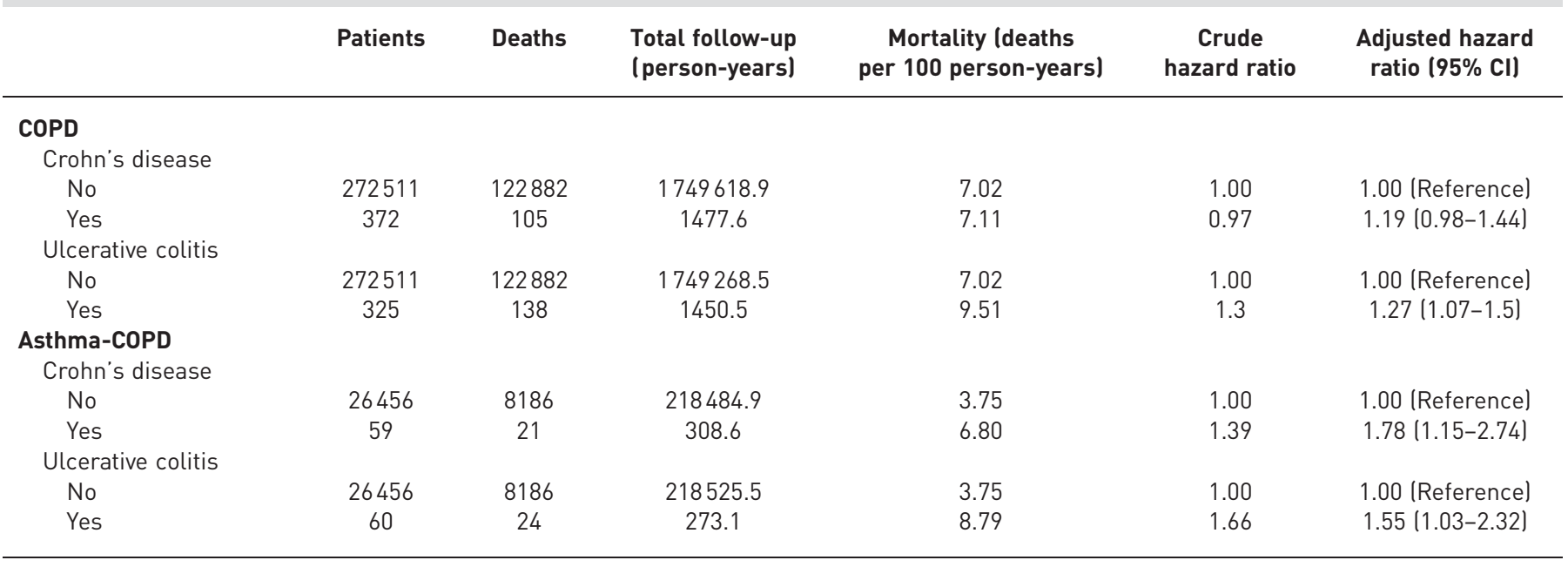

Data are presented as $n$, unless otherwise stated.

asthma-COPD subjects with new-onset IBD was double that observed in subjects without IBD. COPD subjects who developed IBD had a four-fold increase in risk of mortality due to digestive diseases compared with COPD subjects without IBD. These are novel findings that underscore the importance of IBD assessment in patients with COPD who present with digestive symptoms indicative of IBD, such as diarrhoea (even low grade), changes in bowel function, rectal bleeding, abdominal pain, unexplained weight loss, anal fissures or fistula.

Although IBD was shown to be more prevalent among COPD patients [12, 13], and our previous work indicated that IBD can occur in COPD patients even in older age groups regardless of smoking history [11], the impact of developing IBD on important outcomes, such as mortality, has not been assessed.

The reviews of the underlying mechanisms that can explain the association between the two chronic inflammatory conditions point towards systemic inflammation and immunological dysfunctions [9, 10] triggered by environmental factors that IBD and COPD seem to share, such as smoking [25, 26] or air pollution [27, 28]. Furthermore, pulmonary involvement [29-31] is a common extra-intestinal

TABLE 4 Crude and adjusted hazard ratios for the association between inflammatory bowel disease (IBD) and cause-specific mortality in chronic obstructive pulmonary disease patients

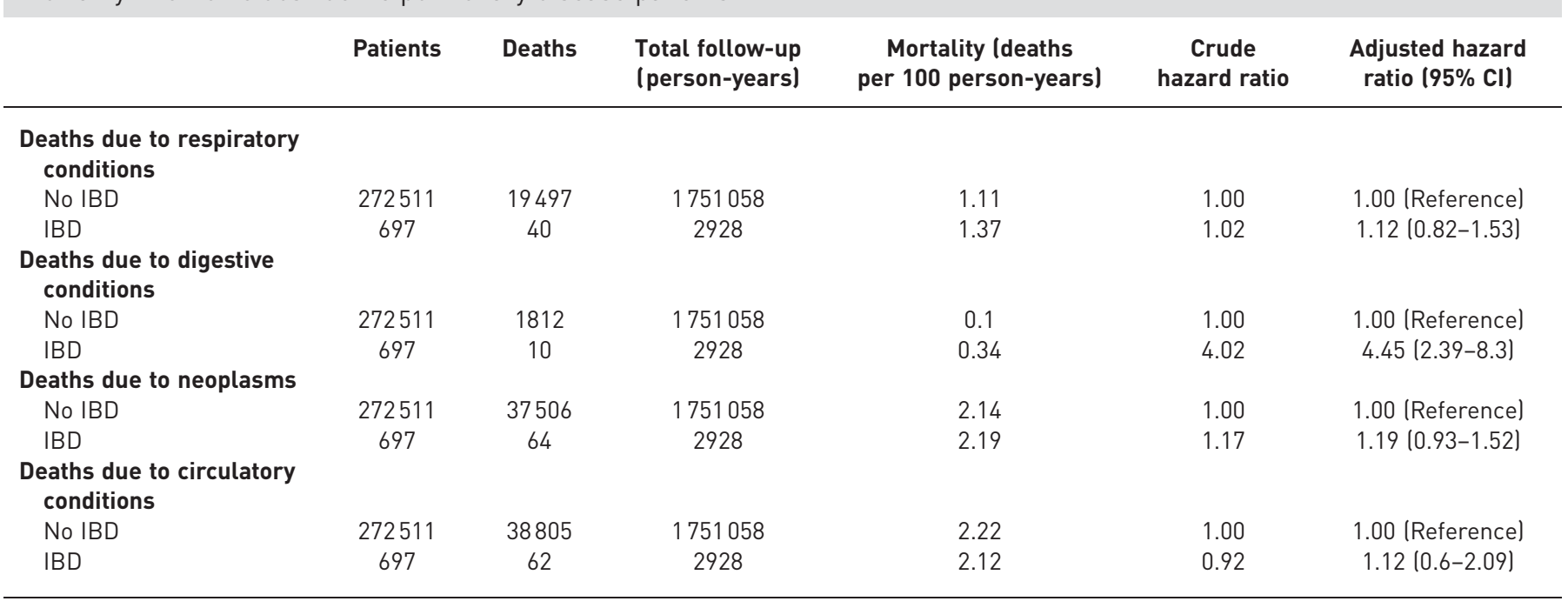

Data are presented as $n$, unless otherwise stated. 
TABLE 5 Crude and adjusted hazard ratios for the association between inflammatory bowel disease (IBD) and cause-specific mortality in asthma-associated chronic obstructive pulmonary disease patients

\begin{tabular}{|c|c|c|c|c|c|}
\hline nts & Deaths & $\begin{array}{l}\text { Total follow-up } \\
\text { (person-years) }\end{array}$ & $\begin{array}{c}\text { Mortality (deaths } \\
\text { per } 100 \text { person-years) }\end{array}$ & $\begin{array}{c}\text { Crude } \\
\text { hazard ratio }\end{array}$ & $\begin{array}{l}\text { Adjusted hazard } \\
\text { ratio }(95 \% \mathrm{CI})\end{array}$ \\
\hline
\end{tabular}

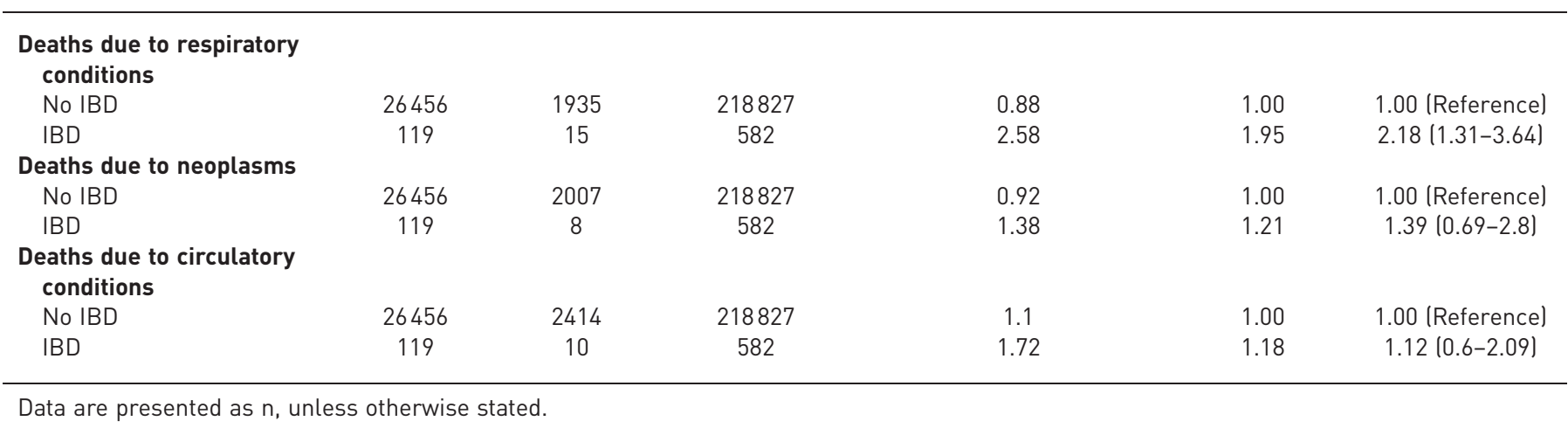

manifestation of IBD and an increased intestinal permeability, one of the factors that may increase susceptibility to develop IBD, was described in COPD patients [32].

The increase observed in digestive mortality in COPD may be due to the fact that IBD itself is associated with increases in mortality, including mortality by digestive conditions [14, 33-36]. The four-fold increase in digestive mortality may have also driven the observed rise in all-cause mortality in COPD. In patients with asthma-COPD, the presence of IBD was associated with a two-fold increase in mortality due to respiratory conditions. There is yet insufficient evidence as to whether IBD may lead to alterations in pulmonary function [30] that could result in disease exacerbations and an increased number of deaths. However, the literature shows that patients presenting with mixed features of asthma and COPD have a greater impairment in lung function [37], experience more severe and more frequent exacerbations than those with COPD alone [38, 39], and have a higher mortality rate [40]. Further studies are needed to confirm whether the observed increase in risk of mortality may be due to the differences in disease severity between COPD and asthma-COPD patients.

Our study has limitations. One may result from the selection of subjects into the COPD and asthma-COPD cohorts. In the past decade a growing number of studies have addressed the asthma-COPD overlap syndrome (ACOS), a clinical entity grouping patients who present with mixed asthma and COPD features. There is no consensus yet regarding a clear clinical definition for ACOS [41] and no validated algorithm recommended for studies based on administrative claims databases. Our methodological approach to differentiate between COPD subjects and those with a mix of asthma and COPD followed the work of BLANCHETTE et al. [38], who classified as ACOS all subjects with claims for both asthma and COPD. We have extended these criteria to include in the asthma-COPD cohort all subjects for whom prescriptions were dispensed for asthma-specific medications. While it is not possible to ascertain what proportion of the asthma-COPD cohort would fulfil the clinical criteria for ACOS, our approach has increased the likelihood that the COPD cohort was cleared of any subjects that may have had associated asthma.

As for most studies based on administrative data, the lack of information regarding smoking is an important limitation. However, previous studies have shown that the IBD association with COPD extends to ulcerative colitis patients as well $[11,13]$. Tobacco smoking plays a protective role in ulcerative colitis [25]; therefore, it is unlikely that confounding by smoking can explain the observed association between IBD and COPD.

In this study we used a population-based cohort design that facilitates the assessment of outcomes of clinical importance, such as mortality, even in rare conditions. The use of a 2-year IBD-free interval prior to the first diagnostic code contributing to the IBD patient definition increased the probability that only incident IBD subjects were included in the analysis. The models were adjusted for the number of hospitalisations and the medication dispensed in the year prior to cohort entry to account for the impact of disease severity and comorbid conditions.

In conclusion, this study further substantiates the previously described association between IBD and COPD, and indicates that IBD could be one of the comorbidities contributing to the already high and increasing COPD mortality. Our findings highlight the importance of awareness of the IBD-COPD association and of IBD assessment in COPD subjects who present with digestive symptoms indicative of 
IBD. Failure to perform an IBD assessment in these patients could lead to delays in diagnosis and treatment, and to an increase in number of complications. Patients with associated asthma-COPD features, in particular, should be carefully evaluated, as there is evidence suggesting they are more severely ill and, therefore, more susceptible to the impact of comorbid conditions than patients with COPD alone.

\section{References}

1 Bousquet J, Khaltaev N. Global Surveillance, Prevention and Control of Chronic Respiratory Diseases: A Comprehensive Approach. Geneva, World Health Organization, 2007.

2 Mathers CD, Loncar D. Projections of global mortality and burden of disease from 2002 to 2030. PLoS Med 2006 3: e442.

3 Ma J, Ward EM, Siegel RL, et al. Temporal trends in mortality in the United States, 1969-2013. JAMA 2015; 314: 1731-1739.

4 Decramer M, Rennard S, Troosters $\mathrm{T}$, et al. COPD as a lung disease with systemic consequences - clinical impact, mechanisms, and potential for early intervention. COPD 2008; 5: 235-256.

5 Gibson PG, McDonald VM. Asthma-COPD overlap 2015: now we are six. Thorax 2015; 70: 683-691.

6 Barrecheguren M, Esquinas C, Miravitlles M. The asthma-chronic obstructive pulmonary disease overlap syndrome (ACOS): opportunities and challenges. Curr Opin Pulm Med 2015; 21: 74-79.

7 Louie S, Zeki AA, Schivo $M$, et al. The asthma-chronic obstructive pulmonary disease overlap syndrome: pharmacotherapeutic considerations. Expert Rev Clin Pharmacol 2013; 6: 197-219.

8 Sin DD, Anthonisen NR, Soriano JB, et al. Mortality in COPD: role of comorbidities. Eur Respir J 2006; 28 : $1245-1257$.

9 Keely S, Talley NJ, Hansbro PM. Pulmonary-intestinal cross-talk in mucosal inflammatory disease. Mucosal Immunol 2012; 5: 7-18.

10 Wang H, Liu JS, Peng SH, et al. Gut-lung crosstalk in pulmonary involvement with inflammatory bowel diseases. World J Gastroenterol 2013; 19: 6794-6804.

11 Brassard P, Vutcovici M, Ernst P, et al. Increased incidence of inflammatory bowel disease in Quebec residents with airway diseases. Eur Respir J 2015; 45: 962-968.

12 Raj AA, Birring SS, Green R, et al. Prevalence of inflammatory bowel disease in patients with airways disease. Respir Med 2008; 102: 780-785.

13 Ekbom A, Brandt L, Granath F, et al. Increased risk of both ulcerative colitis and Crohn's disease in a population suffering from COPD. Lung 2008; 186: 167-172.

14 Bitton A, Vutcovici M, Sewitch M, et al. Mortality trends in Crohn's disease and ulcerative colitis: a population-based study in Quebec, Canada. Inflamm Bowel Dis 2015; 22: 416-423.

15 Jess T, Loftus EV Jr, Harmsen WS, et al. Survival and cause specific mortality in patients with inflammatory bowel disease: a long term outcome study in Olmsted County, Minnesota, 1940-2004. Gut 2006; 55: 1248-1254.

16 Tamblyn R, Lavoie G, Petrella L, et al. The use of prescription claims databases in pharmacoepidemiological research: the accuracy and comprehensiveness of the prescription claims database in Québec. J Clin Epidemiol 1995; 48: 999-1009.

17 Brassard P, Suissa S, Kezouh A, et al. Inhaled corticosteroids and risk of tuberculosis in patients with respiratory diseases. Am J Respir Crit Care Med 2011; 183: 675-678.

18 Ernst P, Baltzan M, Deschênes J, et al. Low-dose inhaled and nasal corticosteroid use and the risk of cataracts. Eur Respir J 2006; 27: 1168-1174.

19 Ernst P, Gonzalez AV, Brassard P, et al. Inhaled corticosteroid use in chronic obstructive pulmonary disease and the risk of hospitalization for pneumonia. Am J Respir Crit Care Med 2007; 176: 162-166.

20 Gonzalez AV, Li G, Suissa S, et al. Risk of glaucoma in elderly patients treated with inhaled corticosteroids for chronic airflow obstruction. Pulm Pharmacol Ther 2010; 23: 65-70.

21 Suissa S, Baltzan M, Kremer R, et al. Inhaled and nasal corticosteroid use and the risk of fracture. Am J Respir Crit Care Med 2004; 169: 83-88.

22 Suissa S, Patenaude V, Lapi F, et al. Inhaled corticosteroids in COPD and the risk of serious pneumonia. Thorax 2013; 68: 1029-1036.

23 Rezaie A, Quan H, Fedorak RN, et al. Development and validation of an administrative case definition for inflammatory bowel diseases. Can J Gastroenterol 2012; 26: 711-717.

24 Bitton A, Vutcovici M, Patenaude V, et al. Epidemiology of inflammatory bowel disease in Quebec: recent trends. Inflamm Bowel Dis 2014; 20: 1770-1776.

25 Birrenbach T, Böcker U. Inflammatory bowel disease and smoking. A review of epidemiology, pathophysiology, and therapeutic implications. Inflamm Bowel Dis 2004; 10: 848-859.

26 Salvi S. Tobacco smoking and environmental risk factors for chronic obstructive pulmonary disease. Clin Chest Med 2014; 35: 17-27.

27 Schikowski T, Mills IC, Anderson HR, et al. Ambient air pollution: a cause of COPD? Eur Respir J 2014; 43: 250-263.

28 Kaplan GG, Hubbard J, Korzenik J, et al. The inflammatory bowel diseases and ambient air pollution: a novel association. Am J Gastroenterol 2010; 105: 2412-2419.

29 Betancourt SL, Palacio D, Jimenez CA, et al. Thoracic manifestations of inflammatory bowel disease. AJR Am J Roentgenol 2011; 197: W452-W456.

30 Black H, Mendoza M, Murin S. Thoracic manifestations of inflammatory bowel disease. Chest 2007; 131: 524-532.

31 Camus P, Piard F, Ashcroft T, et al. The lung in inflammatory bowel disease. Medicine 1993; 72: 151-183.

32 Rutten EP, Lenaerts K, Buurman WA, et al. Disturbed intestinal integrity in patients with COPD: effects of activities of daily living. Chest 2014; 145: 245-252.

33 Hutfless SM, Weng X, Liu L, et al. Mortality by medication use among patients with inflammatory bowel disease, 1996-2003. Gastroenterology 2007; 133: 1779-1786.

34 Jussila A, Virta LJ, Pukkala E, et al. Mortality and causes of death in patients with inflammatory bowel disease: a nationwide register study in Finland. J Crohns Colitis 2014; 8: 1088-1096. 
35 Wolters FL, Russel MG, Sijbrandij J, et al. Disease outcome of inflammatory bowel disease patients: general outline of a Europe-wide population-based 10-year clinical follow-up study. Scand J Gastroenterol Suppl 2006; 243: 46-54.

36 Bernstein CN, Nugent Z, Targownik LE, et al. Predictors and risks for death in a population-based study of persons with IBD in Manitoba. Gut 2015; 64: 1403-1411.

37 Zeki AA, Schivo M, Chan A, et al. The asthma-COPD overlap syndrome: a common clinical problem in the elderly. J Allergy (Cairo) 2011; 2011: 861926.

38 Blanchette CM, Gutierrez B, Ory C, et al. Economic burden in direct costs of concomitant chronic obstructive pulmonary disease and asthma in a Medicare Advantage population. J Manag Care Pharm 2008; 14: 176-185.

39 Hardin M, Silverman EK, Barr RG, et al. The clinical features of the overlap between COPD and asthma. Respir Res 2011; 12: 127.

40 Diaz-Guzman E, Khosravi M, Mannino DM. Asthma, chronic obstructive pulmonary disease, and mortality in the U.S. population. COPD 2011; 8: 400-407.

41 Bujarski S, Parulekar AD, Sharafkhaneh A, et al. The asthma COPD overlap syndrome (ACOS). Curr Allergy Asthma Rep 2015; 15: 509. 